\title{
The N-terminal domain of Spike protein contributes to antigenicity difference between porcine epidemic diarrhea virus genotypes G1 and G2
}

Qi-long Qiao ${ }^{1}$, Ning Li², Ming-zhen Song ${ }^{1}$, Jing Chen ${ }^{1}$, Pan pan Yang ${ }^{2}$, Yu-he Miao ${ }^{3}$, and JUN ZHAO ${ }^{1}$

${ }^{1}$ Henan Agricultural University

${ }^{2}$ Affiliation not available

${ }^{3}$ Fujian Shengwei Biotech Co., Ltd

March 1, 2021

\begin{abstract}
Porcine epidemic diarrhea virus (PEDV) strains have been clarified into two genotypes, G1 and G2, based on the sequence of the spike (S) gene. Amino acid mutations that distinguish the two PEDV genotypes were mostly located in the N-terminal domain (NTD) (aa 1-380) of S protein. The fact of increased outbreaks of G2 subtype PEDV and the failure of G1 subtype PEDV strain (CV777)-based vaccine in China since 2010 suggested that multiple amino acid mutations located in the NTD altered the antigenicity of S protein. To determine the role of the NTD of S protein in the antigenicity difference, the NTD of the CV777 vaccine strain (G1) and CH/ZMDZY/11 strain (G2) was expressed in E. coli, respectively. polyclonal antibodies (PAbs) against genotype-specific $S$ proteins were prepared by immunizing BALB/c mice using purified S proteins. Antigenicity was systematically compared by detection of PAbs against two genotype PEDV strains and purified S proteins using Western blot, indirect enzyme-linked immunosorbent assay (ELISA), indirect immunofluorescence assay (IFA), and serum cross-neutralization assay (SN). Consistent with the multiple amino acid mutations in the NTD of S protein, different antigenic cross-reactivity between the two genotypes was demonstrated. There was six-fold and more than twenty-fold difference in ELISA and SN titer between anti-CV777 S protein antibodies against G1 and G2 subtype strains, respectively. There was twofold and eight-fold difference in ELISA and SN titer between anti-ZMDZY S protein antibodies against G1 and G2 genotype strains, respectively. The results proved that the NTD of S protein contributes to the antigenicity difference between PEDV genotypes G1 and G2, and highlighted a G2 strain should be used to develop a vaccine for providing better protection against prevalent genotype of PEDV.
\end{abstract}

The N-terminal domain of Spike protein contributes to antigenicity difference between porcine epidemic diarrhea virus genotypes G1 and G2

Running title: NTD of S contributes to PEDV antigenic difference

Qi-long Qiao ${ }^{1 \#}$, Ning Li" ${ }^{1 \#}$, Ming-zhen Song ${ }^{1}$, Jing Chen ${ }^{1}$, Pan-Pan Yang ${ }^{2}$, Yu-he Miao ${ }^{*}$, Jun Zhao $^{1 *}$

${ }^{1}$ College of Veterinary Medicine, Henan Agricultural University, 95 Wenhua Road, Zhengzhou, 450002, China

${ }^{2}$ Fujian Shengwei Biotech Co., Ltd, No. A02-3 Jinling Industrial Park, Guangze County, Nanping, Fujian, 354100, China

Correspondence

J. Zhao, College of Animal Science and Veterinary Medicine, Henan Agricultural University; Zhengzhou, China 
Email: zhaoj@henau.edu.cn

\# These authors contributed equally to this work.

\section{Summary}

Porcine epidemic diarrhea virus (PEDV) strains have been clarified into two genotypes, G1 and G2, based on the sequence of the spike (S) gene. Amino acid mutations that distinguish the two PEDV genotypes were mostly located in the N-terminal domain (NTD) (aa 1-380) of S protein. The fact of increased outbreaks of G2 subtype PEDV and the failure of G1 subtype PEDV strain (CV777)-based vaccine in China since 2010 suggested that multiple amino acid mutations located in the NTD altered the antigenicity of S protein. To determine the role of the NTD of S protein in the antigenicity difference, the NTD of the CV777 vaccine strain (G1) and CH/ZMDZY/11 strain (G2) was expressed in E. coli, respectively. Polyclonal antibodies (PAbs) against genotype-specific S proteins were prepared by immunizing BALB/c mice using purified S proteins. Antigenicity was systematically compared by detection of PAbs against two genotype PEDV strains and purified S proteins using Western blot, indirect enzyme-linked immunosorbent assay (ELISA), indirect immunofluorescence assay (IFA), and serum cross-neutralization assay (SN). Consistent with the multiple amino acid mutations in the NTD of S protein, different antigenic cross-reactivity between the two genotypes was demonstrated. There was six-fold and more than twenty-fold difference in ELISA and SN titer between anti-CV777 S protein antibodies against G1 and G2 subtype strains, respectively. There was twofold and eight-fold difference in ELISA and SN titer between anti-ZMDZY S protein antibodies against G1 and G2 genotype strains, respectively. The results proved that the NTD of S protein contributes to the antigenicity difference between PEDV genotypes G1 and G2, and highlighted a G2 strain should be used to develop a vaccine for providing better protection against prevalent genotype of PEDV.

Keywords: antigenicity; difference; N-terminal domain; PEDV genotype; spike protein;

\section{INTRODUCTION}

Porcine epidemic diarrhea virus (PEDV), a member of the genusAlphacoronavirus in the family Coronaviridae of the orderNidovirales, is a main etiology causing porcine epidemic diarrhea (PED) which is a devastating disease characterized by acute watery diarrhea, vomiting, dehydration, and high mortality in neonatal piglets. PEDV was first reported in England and Belgium (Wood, 1977, Pensaert and de Bouck, 1978) in the late 1970s. Outbreaks of PED in China were sporadic (Chen et al., 2010) until late 2010, when PEDV variant strains-caused PED broke out and spread throughout the country (Wang et al., 2013; Sun et al., 2012). PEDV has also been reported in the US (Stevenson et al., 2013) and other countries in North America, including Canada and Mexico (Mole, 2013, Ojkic et al., 2015, Trujillo-Ortega et al., 2016). PEDV has caused considerable economic damage to the global pig industry.

The PEDV genome is a single-stranded positive -sense RNA with approximately $28 \mathrm{~kb}$ in length, and encodes four structural proteins which include spike (S), nucleocapsid (N), envelop (E), and membrane (M) protein. Among these proteins, the $\mathrm{S}$ protein is on the surface of the PEDV virion that plays a pivotal role in regulating interactions with specific host cell receptors to mediate viral entry, inducing neutralizing antibodies in the natural host and determining antigenicity and virulence (Li et al., 2020, Suzuki et al., 2018, Lv et al., 2016, Cruz et al, 2008, Sun et al.,2008, Park et al., 2007) . The S protein is often used to evaluate the genetic diversity of PEDV strains (Li et al., 2012; Lee et al.,2014). Based on the phylogenetic analysis of the S gene, the current reported PEDV strains could be genetically divided into two genotypes: G1 and G2. PEDV G1 and G2 can be further divided into G1a, G1b, and G2a, G2b subtypes, respectively. The G1 contains classical strains, including the prototype strain CV777 and historical vaccine strains. The G2 contains the post-2010 global epidemic isolates (Jung at al., 2020, Won et al., 2020).

The fact of increased outbreaks of G2 PEDV and the failure of G1 PEDV strain (CV777)-based vaccine in China since 2010 implied that the difference of antigenicity between G1 and G2 PEDV strains. Identification of factors contributing to the antigenicity difference between G1 and G2 PEDV strains will lead to the development of an effective PEDV vaccine with better protection against prevalent genotype of PEDV. As 
amino acid mutations that distinguish the two PEDV genotypes were mostly located in the N-terminal domain (NTD) (aa 1-380) of S protein, the objective of this study is to determine the role of the NTD of S protein in resulting antigenicity difference between PEDV G1 and G2. The NTD of the S protein from CV777 vaccine strain (G1) and CH/ZMDZY/11 strain (G2) was expressed in E. coli , respectively. Polyclonal antibodies (PAbs) against genotype-specific S proteins were prepared by immunizing BALB/c mice using purified S proteins. Antigenicity was systematically compared by detection of PAbs against two genotype PEDV strains and purified S proteins using Western blot, indirect enzyme-linked immunosorbent assay (ELISA), indirect immunofluorescence assay (IFA), and serum cross-neutralization assay (SN).

\section{MATERIALS AND METHODS}

\subsection{Cells, virus, bacteria and expression vector}

Vero cells (ATCC CCL-81) were cultured in Dulbecco's modified Eagle's medium (DMEM; Life Technologies, NY, USA) supplemented with $10 \%$ fetal bovine serum (FBS; Life Technologies), $100 \mathrm{U} / \mathrm{ml}$ penicillin G and $100 \mu \mathrm{g} / \mathrm{ml}$ streptomycin. A G1 PEDV CV777 vaccine strain was kindly provided by Dr. Enjuanes, Department of Molecular and Cell Biology, Campus Universidad Autonoma de Madrid, Madrid, Spain. A G2 PEDV strain $\mathrm{CH} / \mathrm{ZMDZY} / 11$ was isolated from 3-day-old piglets suffering from watery diarrhea and dehydration by our laboratory (Wang et al., 2013). These piglets were generated by CV777vaccinceimmunized sows. pET-28a (+) plasmid was purchased from Novagen (WI, USA). Escherichia coli DH5 $\alpha$ and BL21 (DE3) were used for cloning experiments and as a heterologous expression host respectively.

\subsection{Antigenicity and hydrophilicity analyses of NTD of G1 and G2 PEDV S protein}

Antigenicity and hydrophilicity analyses based on the amino acid sequence from 1 to 380 at N-terminal of S proteins of PEDV Chinese variant and CV777 vaccine strain were carried out by using Protean software of DNAStar Lasergene (DNAStar, Inc., Madison, WI). Antigenic indexes of the N-terminal domains (NTD) were calculated under the Jameson-Wolf method, and hydrophilicity plots were constructed by Kyte-Doolittle method, respectively.

\subsection{Expression and purification of recombinant NTD of S proteins}

According to the sequence of the S gene of PEDV G1 strain CV777 (GenBank accession No. AF353511.1) and G2 strain CH/ZMDZY/11 (GenBank accession No. KC196276.1), the N-terminal (aa 1-380) domain (NTD) gene of CV777 and CH/ZMDZY/11 S gene, which was designated as G1-NTD and G2-NTD was synthesized based on the codon usage of Escherichia coli . Restriction endonucleases sites Nco I and Bam HI were introduced at the 5' and 3' end of the synthesized gene, respectively. The synthesized NTD genes were cloned into same sites of pET28a $(+)$. The resulted plasmid was designated as pET-G1-NTD and pET-G2-NTD.

The expression vector pET-G1-NTD and pET-G2-NTD were introduced intoEscherichia coli. BL21 (DE3) bacterial host. BL21 (DE3) strain harboring expression vector was pre-cultured in $5 \mathrm{~mL}$ of Luria-broth (LB) medium containing $50 \mu \mathrm{g} / \mathrm{mL}$ kanamycin at $37^{\circ} \mathrm{C}$ overnight and was then transferred into $1 \mathrm{~L}$ of LB medium containing $50 \mu \mathrm{g} / \mathrm{mL}$ kanamycin. After the optical density at $600 \mathrm{~nm}$ reached $0.8,1 \mathrm{~mL}$ of $1 \mathrm{M}$ isopropyl-Dthiogalactoside (IPTG) was added to the medium, and the cells were further cultured at $25^{\circ} \mathrm{C}$ overnight. The induced cells were collected by centrifugation. The cells (typically 5-6 g wet weight of cells from one liter of culture) were resuspended in $100 \mathrm{~mL}$ lysis buffer $(10 \mathrm{mM}$ sodium phosphate, $0.15 \mathrm{M} \mathrm{NaCl}, 1 \mathrm{mM} \mathrm{EDTA}, \mathrm{pH}$ 7.2 PBS, containing $1 \%$ Nonidet P-40, 1mM phenylmethylsulfonyl fluoride, 1mM 2-mercaptoethanol, and proteinase inhibitor mixture), and sonicated at $0{ }^{\circ} \mathrm{C}$. The insoluble fraction was removed by centrifugation at $10,000 \mathrm{x}$ g for $30 \mathrm{~min}$ at $4{ }^{\circ} \mathrm{C}$. The supernatants were used to analyze the expression of G1-NTD and G2NTD in sodium dodecyl sulfate polyacrylamide gel electrophoresis (SDS-PAGE). Purification of recombinant G1-NTD and G2-NTD proteins was carried out by using the HisPur Ni-NTA Spin Purification Kit (Thermo Fisher Scientific, MA, USA) according to the instructions of manufacture.

\subsection{Preparation of anti-NTD of S protein polyclonal antibody}


All animal procedures were approved by the Ethics Committee of Henan Agricultural University. Two groups of eight-week-old BALB/c mice were respectively immunized subcutaneously three times, each at 2-week intervals, with $100 \mu \mathrm{g} / \mathrm{mouse}$ of recombinant G1-NTD and G2-NTD formulated in Freund's complete adjuvant for the first time, $100 \mu \mathrm{g} /$ mouse of corresponding recombinant protein formulated in Freund's incomplete adjuvant for the second time, and $100 \mu \mathrm{g} /$ mouse of corresponding recombinant protein for the third time. Two weeks after the third immunization, blood was collected from tail vein of mice from each group and pooled, and serum samples were prepared and store at $-20{ }^{\circ} \mathrm{C}$ until use.

\subsection{Western blot}

Same amount of purified G1-NTD and G2-NTD (200 ng/each) were subjected to $12 \%$ SDS-PAGE and transferred to a nitrocellulose (NC) membrane for $45 \mathrm{~min}$. The $\mathrm{NC}$ membrane was blocked overnight at $4{ }^{\circ} \mathrm{C}$ using 5\% non-fat dry milk in PBS-0.05\% Tween-20 (PBST) followed by incubation with the G1- or G2-NTD antisera (1:2000 dilution in PBST) at $37{ }^{\circ} \mathrm{C}$ for $1 \mathrm{~h}$ with gentle rotating. The membrane was incubated with horseradish peroxidase (HRP)-conjugated goat anti-mouse IgG (1:5000 dilution in PBST) at $37{ }^{\circ} \mathrm{C}$ for $1 \mathrm{~h}$, after complete washing with PBST. The protein bands were visualized using Novex@ ECL Chemiluminescent Substrate Reagent Kit (Thermo Fisher Scientific).

\subsection{ELISA}

Ninety-six well plates were coated with $500 \mathrm{ng} /$ well of purified recombinant G1-NTD and G2-NTD overnight at $4{ }^{\circ} \mathrm{C}$. The coated plates were washed three times with PBST, and blocked with $5 \%$ non-fat dry milk in PBST at $37^{\circ} \mathrm{C}$ for $1 \mathrm{~h}$. The plates were washed three times, and then incubated with G1- and G2-NTD antisera (1:200 dilution in PBST). The plates were incubated at $37^{\circ} \mathrm{C}$ for $1.5 \mathrm{~h}$ and washed three times with PBST, and HRP-conjugated goat anti-mouse IgG (1:5000 dilution in PBST) was added to each well. The plates were incubated t $37^{\circ} \mathrm{C}$ for $1.5 \mathrm{~h}$ and washed as above. Finally, $100 \mu \mathrm{l} 3,3$ ', 5, 5'- tetramethylbenzidine (TMB) was added to each well, and the plates were incubated for additional 15 min at $37{ }^{\circ} \mathrm{C}$ in dark. The reaction was stopped by the addition of $1 \mathrm{~N}$ sulfuric acid. The absorbance was read at a wavelength of 450 $\mathrm{nm}$ (OD450) using Multiskan GO (Thermo Fisher Scientific) ELISA plate reader.

\subsection{Indirect immunofluorescence assay (IFA)}

Vero cells grown in 6-well plates were mock infected or infected with PEDV CH/ZMDZY/11 and CV777 strain at multiplicity of infection (MOI) of 0.1 in infection medium (DMEM supplemented with $0.3 \%$ tryptose phosphate broth, $0.02 \%$ Yeast extract, and $10 \mu \mathrm{g} / \mathrm{ml}$ TPCK-treated trypsin). The infected cells were washed three times with phosphate buffered saline (PBS) and fixed with fixed with $4 \%$ paraformaldehyde for 10 min at room temperature (RT) and permeabilized with $0.2 \%$ Triton X-100 in PBS at RT for 10 min. The cells were blocked with $1 \%$ bovine serum albumin (BSA) in PBS for $30 \mathrm{~min}$ at RT and then respectively incubated with anti-G1-NTD and -G2-NTD sera at $37^{\circ} \mathrm{C}$ for $2 \mathrm{~h}$. After being washed three times in PBS, the cells were incubated for $1 \mathrm{~h}$ at $37^{\circ} \mathrm{C}$ with a goat anti-mouse IgG conjugated to Fluorescein- isothiocyanate (Life Technologies, NY, USA). After washing five times with PBS, the cells staining was visualized using an inverted fluorescence microscope (NikonTS-100, Japan).

\subsection{Focus reduction neutralization assay (FRNT)}

The cross-neutralizing activity against G1 and G2 PEDV strains between the anti-G1-NTD and -G2-NTD serum was evaluated using a focus reduction neutralization test (FRNT) with minor modifications (Vaigya et al., 2010). Initially, $500 \mu \mathrm{l}$ of infection medium-diluted virus stock solution (200 pfu/ml) was mixed with equal volume of twofold serial infection medium-diluted either the polyclonal antisera or the control syngeneic sera, and then incubated for $1 \mathrm{~h}$ at $37^{\circ} \mathrm{C}$. The mixture was added to confluent Vero cells in 6-well plates, which were initially washed twice with infection medium, then incubated for $2 \mathrm{~h}$ at room temperature to allow adsorption of the virus. After incubation, the medium was removed. The infected cells were overlaid with $1 \%$ sodium carboxymethyl cellulose that was dissolved infection medium and incubated for $36 \mathrm{~h}$. The overlaid medium was removed and the cells were fixed as in IFA, and incubated with anti-G1-NTD or -G2-NTD serum for $1 \mathrm{~h}$ at $37^{\circ} \mathrm{C}$. After being washed three times with PBS, the monolayer cells were incubated with 
HRP-conjugated goat anti-mouse IgG (1:1000) for $1 \mathrm{~h}$ at $37^{\circ} \mathrm{C}$. Finally, virus-specific foci were positively stained with 3, 3 - diaminobenzidine (DAB) and counted under microscope. Serum neutralization titers were expressed as the reciprocals of the highest serum dilution resulting in reduction of $50 \%$ focus forming unit.

\subsection{Statistical analysis}

All data analysis was performed with GraphPad Prism 5 software. Data are expressed as the mean \pm standard error. The differences between groups were analyzed using the Multiple $t$ test. Significant differences were determined as ${ }^{*} p<0.05$ (significant) or ${ }^{* *} p<0.01$ (highly significant).

\section{RESULTS}

\subsection{Antigenicity and hydrophilicity analyses of NTD of G1 and G2 PEDV S protein}

Our and others' previous researches have shown that G2 PEDV strains showed insertions and deletions in the S gene, especially in the NTD of the S gene, when compared with G1 PEDV strains. To analyze whether these insertions and deletions result in changes in antigenicity of $S$ protein, comparative antigenicity and hydrophilicity analyses of NTD of G1 and G2 PEDV S protein were carried out. Comparison of antigenic index profiles of the NTD between G1 and G2 PEDV S protein strain indicated that two regions containing amino acid deletions/insertions/mutations exhibited higher degree of antigenic change. One region spans from aa 55 to $70 / 74$, another spans from aa $114 / 118$ to $163 / 166$. Two new potential N-linked glycosylation sites (NST and NAT) were present in the S protein of G2 PEDV strains (Figure 1).

\subsection{Expression and purification of recombinant NTD of G1 and G2 PEDV S protein}

The recombinant plasmids, pET-G1-NTD and pET-G2-NTD were transferred into E. coli BL21 (DE3) host. Recombinant G1-NTD and G2-NTD were expressed with a six histidine tag on the C-terminus, yielding fusion proteins of 383 and 386 aa, which gave bands corresponding to the expected molecular masses of approximately $42.60 \mathrm{kDa}$ and $42.61 \mathrm{kDa}$, respectively. Both the recombinant G1-NTD and G2-NTD were expressed mostly as soluble forms in the cytosolic fraction of E. coli(Figure 2). The recombinant G1-NTD and G2-NTD proteins in the cleared cell lysate can be purified by using the HisPur Ni-NTA Spin Purification Kit (Thermo Fisher Scientific, MA, USA) according to the instructions of manufacture.

\section{Evaluation of cross-reactivity of antibodies against G1 and G2 PEDV S proteins by Western blot}

As shown in Figure 3, under same conditions, the G1-NTD antisera reacted to the G1 NTD protein very well, but hardly to the G2-NTD protein; however, the G2-NTD antisera reacted to both G1 and G2 NTD protein, with to G2 NTD protein better. The results indicated that the NTDs of G1 and G2 PEDV S displayed different antigenicity.

\section{Evaluation of cross-reactivityof antibodies against G1 and G2 PEDV S proteins by ELISA}

The ELISA results also indicated significant different cross-reactivity of antibodies against G1 and G2 PEDV $\mathrm{S}$ proteins. As shown in Figure 4A and 4B, the OD450 values of anti-G1-NTD antibody reacting with the G1-NTD was about six fold of those reacting with the G2-NTD. The OD450 values of anti-G2-NTD antibody reacting with the G2-NTD was about 2-fold of those reacting with the G1-NTD.

\section{Evaluation of cross-reactivity of antibodies against G1 and G2 PEDV strain by IFA}

The cross-reactivity of G1- and G2-NTD antisera against G1 and G2 PEDV strain was evaluated by use of IFA. The IFA results confirmed that the G1- and G2-NTD antisera cross-reacted differently with native S proteins of G1 strain CV777 and G2 PEDV strain CH/ZMDZY/11. The G1-NTD antisera reacted strongly with CV777-infected cells (Figure 5B), but weakly with CH/ZMDZY/11-infected cells (Figure 5F). Conversely, the G2-NTD antisera reacted very strongly with CH/ZMDZY/11-infected cells (Figure 5H), and moderately with CV777-infected cells (Figure 5D). Vero cells infected with G1 and G2 PEDV strains showed distinct morphology of cytopathic effects (CPE). G2 PEDV strain CH/ZMDZY/11-infected cells had higher cell fusion activity than G1 strain CV777. At the same postinoculation time, the CPE and syncytia induced 
by PEDV CH/ZMDZY/11 were much larger than those induced by CV777 vaccine strain. Positive signals of G2 PEDV strain $\mathrm{CH} / \mathrm{ZMDZY} / 11 \mathrm{~S}$ protein antigens were mainly detected as diffuse fluorescence in the center of the syncytia by G1-NTD antisera but as bright and concentrated fluorescence within the cytoplasm of syncytia by G2-NTD antisera.

\section{Evaluation of cross-neutralization of antibodies against G1 and G2 PEDV strain by focus reduction neutralization (FRNT) assay}

As the CPEs induced by G1 and G2 PEDV strain were different, the G1- and G2-NTD antisera were tested for their cross-neutralizing activity against the G1 strain CV777 and G2 strains CH/ZMDZY/11 by a FRNT assay rather than conventional plaque reduction neutralization assay. The PEDV-specific foci were identified by using immuno-peroxidase monolayer assay (IPMA). As shown in Figure 6, the single focus in CV777-infected cells and syncytia focus formed in CH/ZMDZY/11-infected cells could be identified clearly.

As shown in Figure 7A and 7B, infection of the G1 strain CV777 and G2 strain CH/ZMDZY/11 to Vero cells was effectively inhibited by the G1- and G2-NTD antisera respectively. The G1-NTD antisera was highly effective in inhibiting G1 strain CV777 infection in Vero cells with mean NA titers of 172. The mean NA titer of G2-NTD antisera against G2 strain CH/ZMDZY/11 reached 68. Both the mean NA titer of G1-NTD antisera against G2 strain $\mathrm{CH} / \mathrm{ZMDZY} / 11$ and that of G2-NTD antisera against G1 strain CV777 were only 8 (Figure 8). The mean titer of G1-NTD antisera neutralizing G1 strain CV777 was significantly higher than that of cross-neutralizing G2 strain CH/ZMDZY/11 $(p<0.01)$. The mean titer of G2-NTD antisera neutralizing G2 strain CH/ZMDZY/11 was also significantly higher than that of cross-neutralizing G1 strain CV777 $(p<0.01)$

\section{DISCUSSION}

Outbreaks of novel genotype 2 PEDV have resulted in significant economic losses to the swine industry in Asia and North America. All reported PEDV strains have been clarified into two genotypes, G1 and G2, based on the phylogenetic analysis of the $\mathrm{S}$ gene. The $\mathrm{S}$ protein plays important roles in infection and inducing virus neutralizing antibodies ( $\mathrm{Li}$ et al., 2020). The G2 PEDV comprises the post-2010 global epidemic isolates including mutations mainly in the $\mathrm{N}$ terminal domain of S1 (S1-NTD) (Fan et al., 2017, Zhang et al., 2021. These mutations affect the conformational structure and N-linked glycosylation of S1-NTD, which may alter the pathogenicity and antigenicity of the G2 PEDV (Chen et al., 2019, Suzuki et al., 2018). Vaccination is an effective strategy for control and prevention of PED during epidemic or endemic outbreaks. Despite the administration of killed or attenuated vaccines based on G1 PEDV strain CV777, the G2 PEDV outbreaks in vaccinated herds in China still increased dramatically since 2010 (Li et al., 2012). A recent study evaluated the cross-protection between G1a- and G2a-genotype PEDVs in suckling piglets (Zhang et al., 2020). They demonstrated that all piglets from the sows immunized with $\mathrm{CH} / \mathrm{JX} / 01$ (G2) could not only survive when challenged with the homologous PEDV, but also be fully protected when challenged with heterogenous G1 PEDV. However, the piglets from the sows immunized with CV777 (G1) could be protected when challenged with homologous PEDV and only partially protected when challenged with heterologous G2 strain of PEDV (CH/JX/01). This implied that immunization failure of G1 PEDV strain-based vaccines could be due to the mutations in the S gene contributing to the antigenicity difference between G1 and G2 PEDV strains. Identification of the role of the NTD of S protein in antigenicity difference between G1 and G2 PEDV strains will lead to the development of an effective PEDV vaccine with better protection against prevalent genotype of PEDV.

Most of previous S protein functional studies have focused on the core neutralizing epitope (COE), S1, full-length S and S2 proteins (Wang et al., 2016, Oh et al., 2014, Makadiya et al., 2016). To the best of our knowledge, our present study is the first work focused on the contribution of NTD (aa 1-380) of S protein to the antigenicity difference between G1 and G2 PEDV strains. In the present study, A G1 PEDV CV777 vaccine strain and G2 strain CH/ZMDZY/11 isolated from a CV777-immunized herd (Wang et al., 2013). Comparison of antigenic index profiles of the S proteins PEDV G1 strain CV777 and G2 strain $\mathrm{CH} / \mathrm{ZMDZY} / 11$ indicated the most dissimilar regions were in NTD of the S protein, and there were two 
regions in NTD exhibited higher degree of antigenic change than other regions. The aa mutations generated two new N-linked glycosylation sites (NST and NAT) in G2 PEDV strain CH/ZMDZY/11. This is consistent with previous comparison of antigenic index profiles of the NTD of S protein between the prototype strain CV777 and a US strain (Huang et al., 2013). We assumed that vaccines based on the historical CV777 strain became antigenically less related to G2 PEDV variant strains emerged post-2010 in China due to the mutations in the NTD of S protein.

To confirmed this hypothesis, the NTDs of S genes of the G1 PEDV strain CV777 and a G2 PEDV strain $\mathrm{CH} / \mathrm{ZMDZY} / 11$ were expressed in E. coli. , respectively. Cross-reactivity of G1- and G2-NTD antisera against G1 and G2 PEDV strains was evaluated using Western blot, ELISA, IFA, and SN. The Western blot and ELISA results confirmed the antigenicity of the G1- and G2-NTD proteins and the significant difference in cross-reactivity between the NTD proteins of two PEDV genotypes. The IFA results indicated different crossreactivity of G1- and G2-NTD antisera against native S proteins of PEDV. Notably, difference in morphology of cytopathic effects (CPE) was observed in G1 strain CV777 and G2 strain CH/ZMDZY/11 infected cells in IFA. CH/ZMDZY/11-infected cells developed CPE characterized by cell fusion and syncytium. However, CV777-infected cells developed CPE characterized by individual round cell with enlarged nuclei. This implied that mutations in the NTD may alter the pathogenicity of PEDV.

Regarding the effects of amino acid mutations in the NTD on the neutralizing activity of anti-NTD polyclonal antibodies, G1- and G2-NTD antisera also displayed significant differences in SN titers against G1 and G2 PEDV strains. The SN assay indicated antigenic differences of 8- to 20-fold between the two PEDV genotypes. The NA titer of the G1-NTD antisera against G1 CV777 vaccine strain was more than 20-fold higher than that of against G2 CH/ZMDZY/11 strain. Interestingly, the NA titer of G2-NTD antisera against G2 strain $\mathrm{CH} / \mathrm{ZMDZY} / 11$ was only 8-fold higher than that of against G1 strain CV777. This could be due to the two new N-linked glycosylation sites in the NTD of $\mathrm{CH} / \mathrm{ZMDZY} / 11$ strain $\mathrm{S}$ protein. It is believed that glycosylation sites on viral surface glycoproteins play a role in glycan shielding which is a primary mechanism by which the virus evades neutralizing immune responses (Wei et al.,2012). Alteration of a glycosylation site can have dramatic consequences for a virus. It has been demonstrated that alteration of a glycosylation site can impact protein folding and conformation (Hebert et al., 1997) and affect distant parts of a protein through masking or conformational changes. This could result in tighter packing of glycoprotein regions involved in neutralization epitopes, reduce accessibility, and so also facilitate immune escape (Ye et al., 2000).

A previous study showed antigenic differences of twofold between the two PEDV genotypes, in which the immunogenicity and antigenic relationships among full-length S proteins of G1 strain CV777 and G2 strain LNCT2 was investigated (Wang et al.,2016). Neutralizing epitopes reside in other domains of S protein might lower the differences between the two PEDV genotypes. Our results suggested that there were neutralizing epitopes in the NTD (aa 1-380) of the S protein and new neutralizing epitopes had emerged in the post-2010 G2 PEDV variant strains. This result is consistent with a previous study in which potent neutralization was observed with antibodies targeting the NTD (1-220aa) (Li et al., 2017). Sialic acid (Sia) binding activity has been mapped to the amino-terminal 246 residues of the PEDV S protein (Deng et al., 2016, Li et al., 2016, Liu et al., 2015). This suggested that cell attachment domains of PEDV could reside in the NTD of S protein. Cell attachment domains of the S protein are key targets of neutralizing antibodies. Potent neutralization against G1 and G2 PEDV straachieved by antibodies targeting the NTD further underline the importance of NTD of S protein as major targets of potent neutralizing antibodies.

Taken together, our data indicated that the NTD of S protein contributes to the antigenicity difference between G1 and G2 PEDV strains, and the existence of neutralizing epitopes in the NTD of S protein. An ideal candidate strain used for PEDV vaccine is expected to have the ability to confer excellent protection against different field strains of PEDV. Results in the present study also highlights the necessity of choosing currently circulating G2 PEDV for the development of novel PEDV vaccine candidates with improved efficacy.

\section{ACKNOWLEDGMENTS}


The study was supported by the National Key R\&D Program of China (NO 2016YFD0501100).

\section{CONFLICT OF INTEREST}

The authors declare no conflict of interest.

\section{REFERENCES}

Chen, J., Wang, C., Shi, H., Qiu, H., Liu, S., Chen, X., Zhang, Z., Feng, L. (2010). Molecular epidemiology of porcine epidemic diarrhea virus in China. Archives of Virology , 155,14711476.https://doi.org/10.1007/s00705-010-0720-2.

Chen, P., Wang, K., Hou, Y., Li, H., Li, X., Yu, L., Jiang, Y., Gao, F., Tong, W., Yu, H., Yang, Z., Tong, G., Zhou, Y. (2019). Genetic evolution analysis and pathogenicity assessment of porcine epidemic diarrhea virus strains circulating in part of China during 2011-2017. Infection, Genetics and Evolution , 69, 153165.https://doi.org/10.1016/j.meegid.2019.01.022.

Cruz, D. J., Kim, C. J., Shin, H. J. (2008). The GPRLQPY motif located at the carboxy-terminal of the spike protein induces antibodies that neutralize Porcine epidemic diarrhea virus. Virus Research, 32, 192196.https://doi.org/10.1016/j.virusres.2007.10.015.

Deng, F., Ye, G., Liu, Q., Navid, M. T., Zhong, X., Li, Y., Wan, C., Xiao, S., He, Q., Fu, Z. F., Peng, G. (2016). Identification and comparison of receptor binding characteristics of the spike protein of two porcine epidemic diarrhea virus strains. Viruses , 8, 55.https://doi.org/10.3390/v8030055.

Fan, B., Jiao, D., Zhao, X., Pang, F., Xiao, Q., Yu, Z., Mao, A., Guo, R., Yuan, W., Zhao, P., He, K., Li, B. (2017). Characterization of Chinese porcine epidemic diarrhea virus with novel insertions and deletions in genome. Scientific Report, 7, 44209.https://doi.org/10.1038/srep44209.

Hebert, D.N., Zhang, J.X., Chen, W., Foellmer, B., and Helenius, A. (1997). The number and location of glycans on influenza hemagglutinin determine folding and association with calnexin and calreticulin. Journal of Cell Biology, 139, 613-623.https://doi.org/10.1083/jcb.139.3.613.

Huang, Y. W., Dickerman, A. W., Piñeyro, P., Li, L., Fang, L., Kiehne, R., Opriessnig, T., Meng, X. J. (2013). Origin, evolution, and genotyping of emergent porcine epidemic diarrhea virus strains in the United States. mBio. 4, e00737-13.https://doi.org/10.1128/mBio.00737-13.

Jung, K., Saif, L. J., Wang, Q. (2020). Porcine epidemic diarrhea virus (PEDV): An update on etiology, transmission, pathogenesis, and prevention and control. Virus Research , 286, 198045.https://doi.org/10.1016/j.virusres.2020.198045.

Lee, S., Lee, C. (2014). Outbreak-related porcine epidemic diarrhea virus strains similar to US strains, South Korea, 2013. Emerging Infectious Diseases , 20, 1223-1226.https://doi.org/10.3201/eid2007.140294.

Li, C., Li, W., Lucio de Esesarte, E., Guo, H., van den Elzen, P., Aarts, E., van den Born, E., Rottier, P. J. M., Bosch, B. J. (2017). Cell attachment domains of the porcine epidemic diarrhea virus spike protein are key targets of neutralizing antibodies. Journal of Virology, 91, e00273-17.https://doi.org/10.1128/JVI.00273-17.

Li, W., Li, H., Liu, Y., Pan, Y., Deng, F., Song, Y., Tang, X., He, Q. (2012). New variants of porcine epidemic diarrhea virus, China, 2011.Emerging Infectious Diseases , 18,13501353.https://doi.org/10.3201/eid1808.120002.

Li, W., van Kuppeveld, F. J. M., He, Q., Rottier, P. J. M., Bosch, B. J. (2016). Cellular entry of the porcine epidemic diarrhea virus. Virus Research , 226,117-127.https://doi.org/10.1016/j.virusres.2016.05.031.

Li, Z., Ma, Z., Li, Y., Gao, S., Xiao, S. (2020). Porcine epidemic diarrhea virus: Molecular mechanisms of attenuation and vaccines. Microbial Pathogenesis , 149, 104553.https://doi.org/10.1016/j.micpath.2020.104553. 
Liu, C., Tang, J., Ma, Y., Liang, X., Yang, Y., Peng, G., Qi, Q., Jiang, S., Li, J., Du, L., Li, F. (2015). Receptor usage and cell entry of porcine epidemic diarrhea coronavirus. Journal of Virology , 89, 61216125.https://doi.org/10.1128/JVI.00430-15.

Lv, C., Xiao, Y., Li, X., Tian, K. (2016). Porcine epidemic diarrhea virus: Current insights. Virus Adaptation and Treatment, 8, 1-12.https://doi.org/10.2147/VAAT.S107275.

Makadiya, N., Brownlie, R., Van den Hurk, J., Berube, N., Allan, B., Gerdts, V., Zakhartchouk, A. (2016). S1 domain of the porcine epidemic diarrhea virus spike protein as a vaccine antigen. Virology Journal , 13, 57.https://doi.org/10.1186/s12985-016-0512-8.

Mole, B. (2013). Deadly pig virus slips through US borders.Nature , 499, 388.https://doi.org/10.1038/499388a.

Oh, J., Lee, K.W., Choi, H.W., Lee, C. (2014). Immunogenicity and protective efficacy of recombinant S1 domain of the porcine epidemic diarrhea virus spike protein. Archives of Virology , 159, 29772987.https://doi.org/10.1007/s00705-014-2163-7.

Ojkic, D., Hazlett, M., Fairles, J., Marom, A., Slavic, D., Maxie, G., Alexandersen, S., Pasick, J., Alsop, J., Burlatschenko, S. (2015). The first case of porcine epidemic diarrhea in Canada. Canadian Veterinary Journal , 56, 149-152.

Park, S. J., Song, D. S., Ha, G. W., Park, B. K. (2007). Cloning and further sequence analysis of the spike gene of attenuated porcine epidemic diarrhea virus DR13. Virus Genes , 35,55-64.https://doi.org/10.1007/s11262006-0036-1.

Pensaert, M. B., de Bouck, P. (1978). A new coronavirus-like particle associated with diarrhea in swine. Archives of Virology , 58, 243-247.https://doi.org/10.1007/BF01317606.

Stevenson, G. W., Hoang, H., Schwartz, K. J., Burrough, E. R., Sun, D., Madson, D., Cooper, V. L., Pillatzki, A., Gauger, P., Schmitt, B. J., Koster, L. G., Killian, M. L., Yoon, K. J. (2013). Emergence of porcine epidemic diarrhea virus in the United States: clinical signs, lesions, and viral genomic sequences. Journal of Veterinary Diagnostic Investigation, 25, 649-654.https://doi.org/10.1177/1040638713501675.

Sun, D., Feng, L., Shi, H., Chen, J., Cui, X., Chen, H., Liu, S., Tong, Y., Wang, Y., Tong, G. (2008). Identification of two novel B cell epitopes on porcine epidemic diarrhea virus spike protein. Veterinary Microbiology , 131, 73-81.https://doi.org/10.1016/j.vetmic.2008.02.022.

Sun, R. Q., Cai, R. J., Chen, Y. Q., Liang, P. S., Chen, D. K., Song, C. X. (2012). Outbreak of porcine epidemic diarrhea in suckling piglets, China. Emerging Infectious Diseases , 18, 161163.https://doi.org/10.3201/eid1801.111259.

Suzuki, T., Terada, Y., Enjuanes, L., Ohashi, S., Kamitani, W. (2018). S1 subunit of spike protein from a current highly virulent porcine epidemic diarrhea virus is an important determinant of virulence in piglets. Viruses, 10, 467.https://doi.org/10.3390/v10090467.

Trujillo-Ortega, M. E., Beltrán-Figueroa, R., García-Hernández, M. E., Juárez-Ramírez, M., SotomayorGonzález, A., Hernández-Villegas, E. N., Becerra-Hernández, J. F., Sarmiento-Silva, R. E. (2016). Isolation and characterization of porcine epidemic diarrhea virus associated with the 2014 disease outbreak in Mexico: case report. BMC Veterinary Research , 12, 132.https://doi.org/10.1186/s12917-016-0763-z.

Vaidya, S. R., Brown, D. W., Jin, L., Samuel, D., Andrews, N., Brown, K. E. (2010). Development of a focus reduction neutralization test (FRNT) for detection of mumps virus neutralizing antibodies. Journal of Virological Methods, 163, 153-156.https://doi.org/10.1016/j.jviromet.2009.09.006.

Wang, X., Chen, J., Shi, D., Shi, H., Zhang, X., Yuan, J., Jiang, S., Feng, L. (2016). Immunogenicity and antigenic relationships among spike proteins of porcine epidemic diarrhea virus subtypes G1 and G2.Archives of Virology , 161, 537-547.https://doi.org/10.1007/s00705-015-2694-6. 
Wang, X. M., Niu, B. B., Yan, H., Gao, D. S., Yang, X., Chen, L., Chang, H. T., Zhao, J., Wang, C. Q. (2013). Genetic properties of endemic Chinese porcine epidemic diarrhea virus strains isolated since 2010.Archives of Virology , 158, 2487-2494.https://doi.org/10.1007/s00705-013-1767-7.

Wei, Z., Lin, T., Sun, L., Li, Y., Wang, X., Gao, F., Liu, R., Chen, C., Tong, G., Yuan, S. (2012). N-linked glycosylation of GP5 of porcine reproductive and respiratory syndrome virus is critically important for virus replication in vivo. Journal of Virology, 86, 9941-9951.https://doi.org/10.1128/JVI.07067-11.

Won, H., Lim, J., Noh, Y. H., Yoon, I., Yoo, H. S. (2020). Efficacy of porcine epidemic diarrhea vaccines: A systematic review and meta-analysis. Vaccines(Basel) , 8, 642.https://doi.org/10.3390/vaccines8040642

Wood, E. N. (1977). An apparently new syndrome of porcine epidemic diarrhoea. Veterinary Record , 100, 243-244.https://doi.org/10.1136/vr.100.12.243.

Ye, Y., Si, Z.H., Moore, J.P., and Sodroski, J. (2000). Association of structural changes in the V2 and V3 loops of the gp120 envelope glycoprotein with acquisition of neutralization resistance in a simian-human immunodeficiency virus passaged in vivo. Journal of Virology , 74,1195511962.https://doi.org/10.1128/jvi.74.24.11955-11962.2000.

Zhang, H., Han, F., Yan, X., Liu, L., Shu, X., Hu, H. (2021). Prevalence and phylogenetic analysis of spike gene of porcine epidemic diarrhea virus in Henan province, China in 2015-2019. Infection, Genetics and Evolution, 88, 04709.https://doi.org/10.1016/j.meegid.2021.104709.

Zhang, Y., Chen, Y., Yuan, W., Peng, Q., Zhang, F., Ye, Y., Huang, D., Ding, Z., Lin, L., He, H., Wu, Q., Song, D., Tang, Y. (2020). Evaluation of cross-protection between G1a- and G2a-Genotype porcine epidemic diarrhea viruses in suckling piglets. Animals (Basel), 10,1674.https://doi.org/10.3390/ani10091674.

\section{FIGURE LEGENDS}

FIGURE 1 . Comparison of antigenic index profiles of the N-terminal domain (aa 1-380) of the S protein between PEDV genotype 1 CV777 vaccine strain and genotype $2 \mathrm{CH} / \mathrm{ZMDZY} / 11$ strain. Antigenic index was calculated using Protean of DNAStar Lasergene under the Jameson-Wolf method. The corresponding alignment of amino acid sequences and positions of the two regions containing amino acid mutations, deletions, and insertions are shown. Mismatched amino acids are marked in red. Insertions are marked in blue and italic. Deletions are indicated by dashes. The predicated N-linked glycosylation sites are underlined.

FIGURE 2 . Expression and purification of recombinant N-terminal domain (NTD) of S protein of G1 and G2 PEDV strain. M. Protein Marker; 1. pET28a-G2-NTD/BL21 uninduced; 2. pET28a-G2-NTD/BL21 induced; 3. Purified G2-NTD protein; 4. pET28a-G1-NTD/BL21 uninduced; 5. pET28a-G1-NTD/BL21 induced; 6. Purified of G1-NTD protein

FIGURE 3. Western blot analysis of cross-reactivity of antibodies against G1 and G2 PEDV S proteins (A) Same amount of recombinant G1-NTD and G2-NTD was loaded onto SDS-PAGE gel; (B) G1-NTD antisera was used as the primary antibody; (C) G2-NTD antisera was used as the primary antibody.

FIGURE 4 . ELISA analysis of cross-reactivity of antibodies against G1 and G2 PEDV S proteins. (A) G1-NTD antisera was used as the primary antibody; (B) G2-NTD antisera was used as the primary antibody.

FIGURE 5. Evaluation of cross-reactivity of antibodies against G1 and G2 PEDV strain by indirect immunofluorescence assay. Vero cell monolayers were infected with the PEDV G1 CV777 vaccine strain and G2 CH/ZMDZY/11 strain, respectively. At $36 \mathrm{~h}$ postinfection, cytopathic effects were recorded and cells were examined by IFA using anti-S antibodies. G1-NTD antisera (B and F) and G2-NTD antisera (D and $\mathrm{H}$ ) were used as the primary antibody, respectively. All images were taken at a 100-fold magnification. The syncytia induced by the $\mathrm{CH} / \mathrm{ZMDZY} / 11$ (E to $\mathrm{H}$ ) were indicated by arrows.

FIGURE 6 . Foci staining in Vero cell infected with PEDV G1 CV777 vaccine strain and G2 CH/ZMDZY/11 strain by using IPMA in focus reduction neutralization assay. At $36 \mathrm{~h}$ postinfection, foci were detected by 
IPMA using anti-S antibodies as the primary antibody and HRP-labelled anti-mouse IgG as the secondary antibody.

FIGURE 7. Evaluation of cross-neutralization of antibodies against G1 and G2 PEDV strain by focus reduction neutralization assay. (A) Cross-neutralization of G1-NTD antisera against G1 and G2 PEDV strains; (B) Cross-neutralization of G2-NTD antisera against G1 and G2 PEDV strains; Neg. Negative serum; blank. Medium control.

FIGURE 8. Reciprocal of PEDV neutralizing antibody titers of G1- and G2-NTD antisera against the CV777 vaccine and $\mathrm{CH} / \mathrm{ZMDZY} / 11$ strains determined by focus reduction neutralization assay. The virus neutralization antibody titer of each serum was expressed as reciprocal of serum dilution giving $50 \%$ reduction in the number of focus forming unit. Values are representative of the mean from three independent experiments in the duplicate and error bars denote standard deviations.
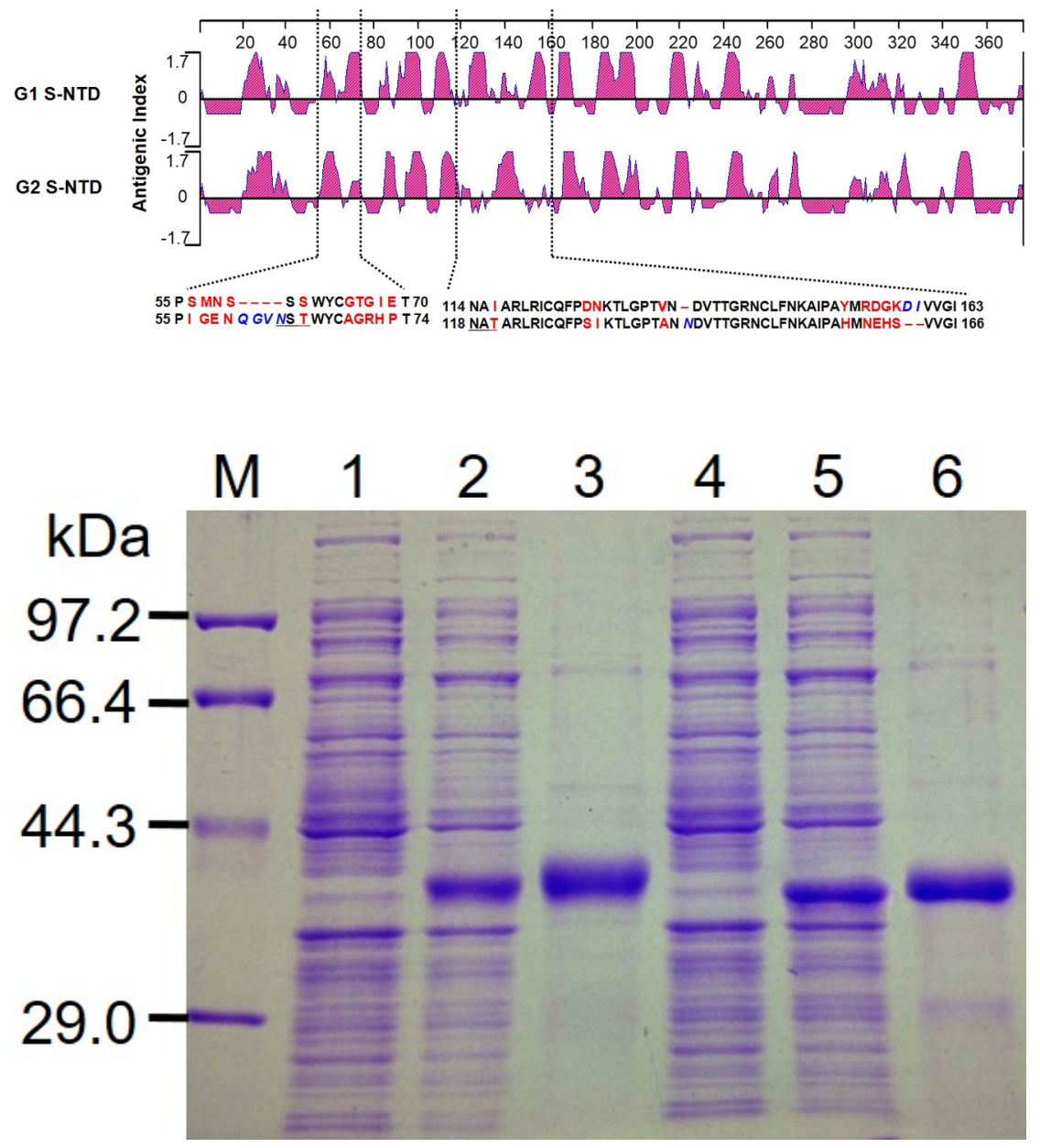

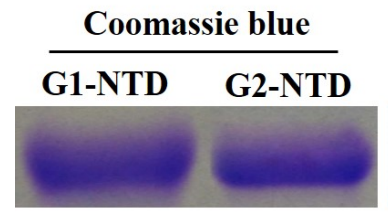

A

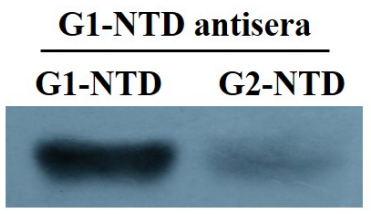

B

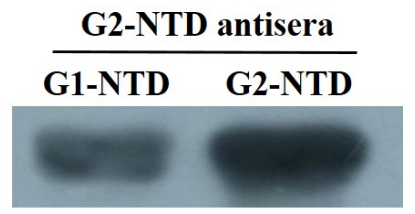

C 
A

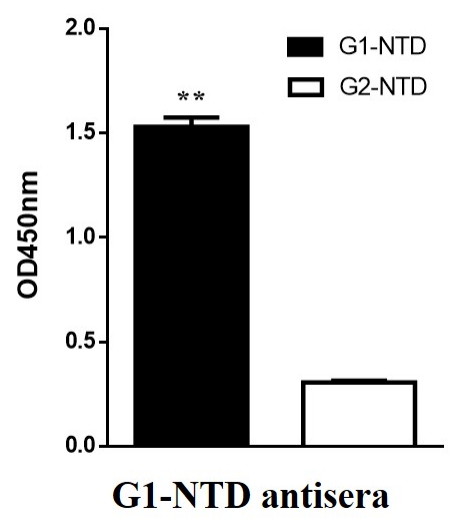

B

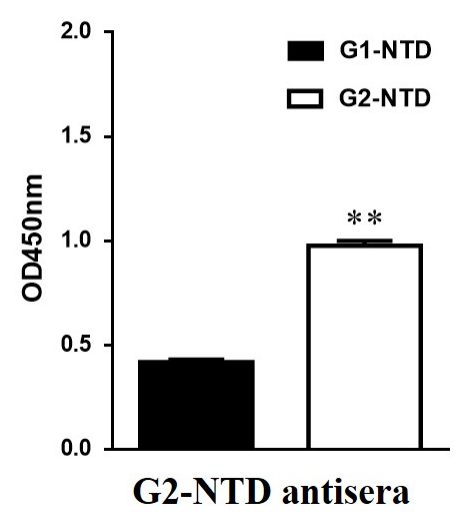

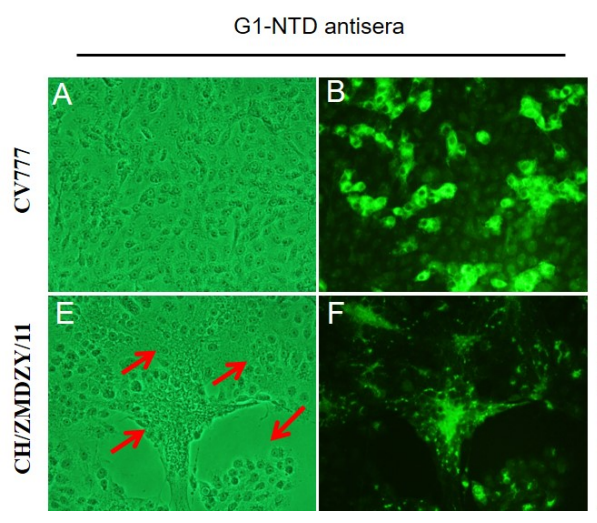
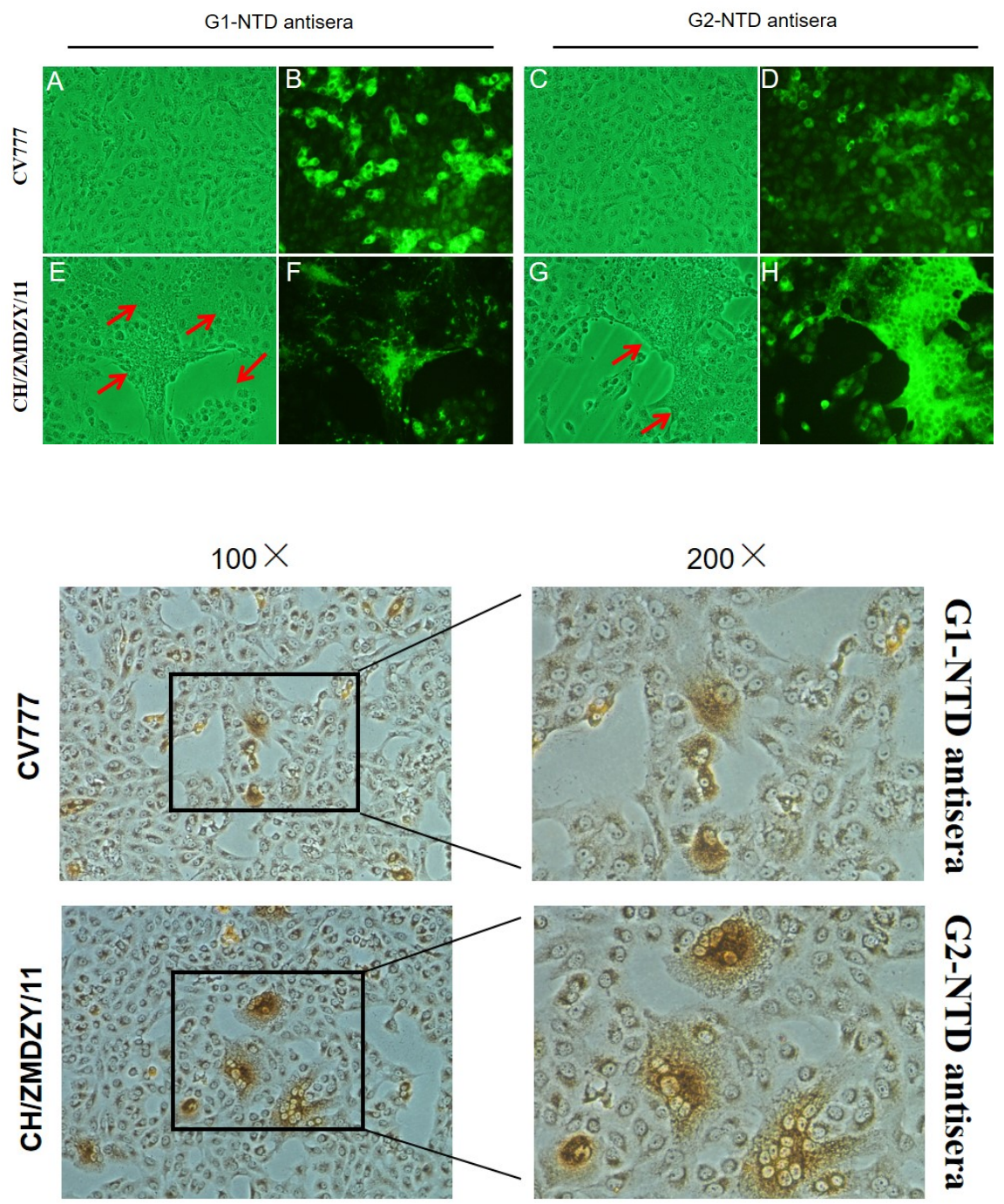

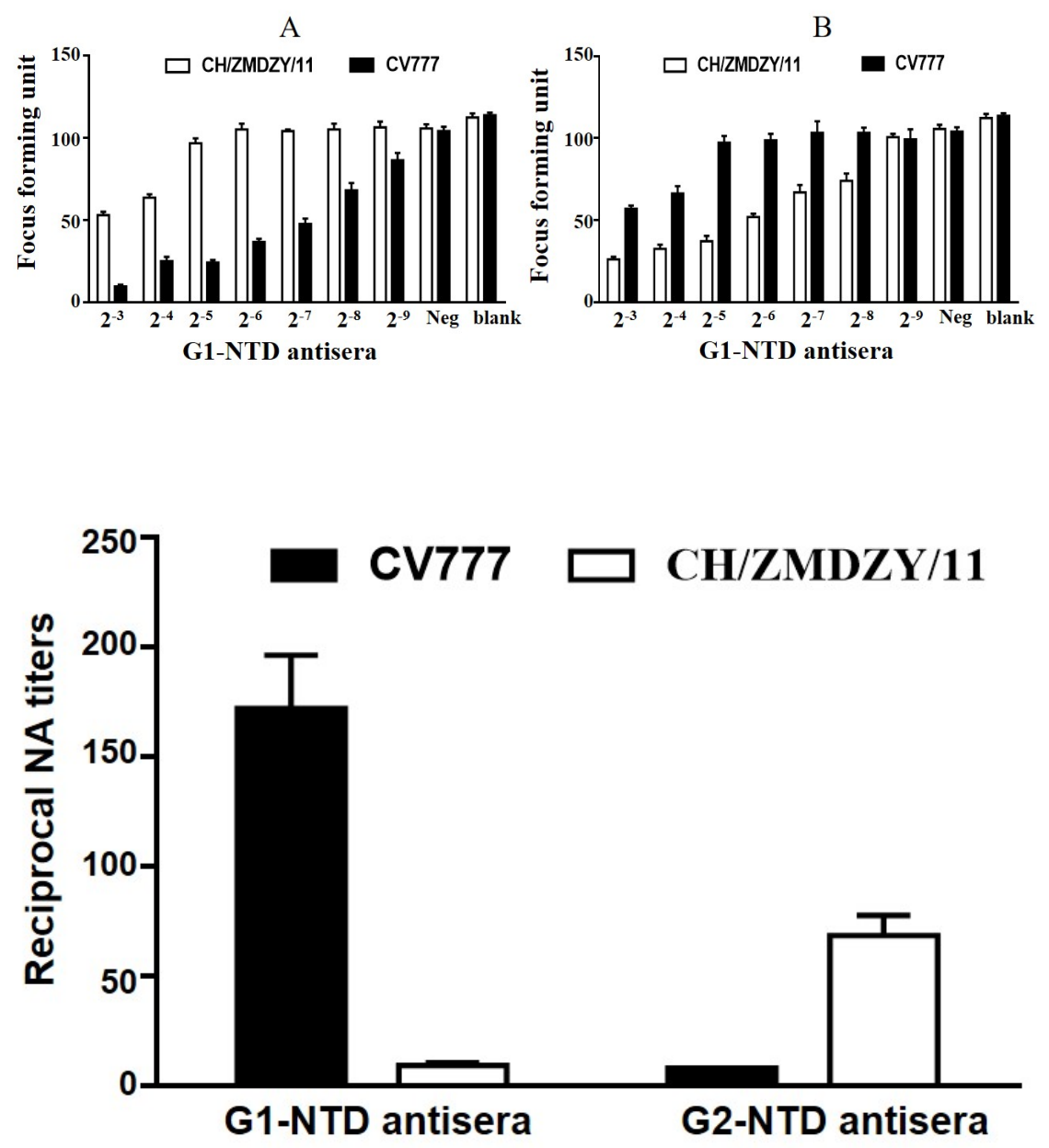